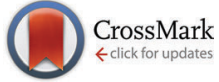

Cite this: Phys. Chem. Chem. Phys., $2017,19,6233$

Received 27th December 2016, Accepted 24th January 2017

DOI: $10.1039 / c 6 c p 08851 a$

rsc.li/pccp

\section{Erbium ion implantation into diamond - measurement and modelling of the crystal structure $\dagger$}

\author{
Jakub Cajzl, *ab Pavla Nekvindová, ${ }^{a}$ Anna Macková, ${ }^{\text {cd }}$ Petr Malinský, ${ }^{\text {cd }}$ \\ David Sedmidubský, ${ }^{a}$ Michal Hušák, ${ }^{a}$ Zdeněk Remeš, ${ }^{e}$ Marián Varga, ${ }^{e}$ \\ Alexander Kromka, ${ }^{e}$ Roman Böttger ${ }^{f}$ and Jiři Oswald ${ }^{e}$
}

\begin{abstract}
Diamond is proposed as an extraordinary material usable in interdisciplinary fields, especially in optics and photonics. In this contribution we focus on the doping of diamond with erbium as an optically active centre. In the theoretical part of the study based on DFT simulations we have developed two Er-doped diamond structural models with 0 to 4 carbon vacancies in the vicinity of the $\mathrm{Er}$ atom and performed geometry optimizations by the calculation of cohesive energies and defect formation energies. The theoretical results showed an excellent agreement between the calculated and experimental cohesive energies for the parent diamond. The highest values of cohesive energies and the lowest values of defect formation energies were obtained for models with erbium in the substitutional carbon position with 1 or 3 vacancies in the vicinity of the erbium atom. From the geometry optimization the structural model with 1 vacancy had an octahedral symmetry whereas the model with 3 vacancies had a coordination of 10 forming a trigonal structure with a hexagonal ring. In the experimental part, erbium doped diamond crystal samples were prepared by ion implantation of $\mathrm{Er}^{+}$ions using ion implantation fluences ranging from $1 \times 10^{14}$ ions per $\mathrm{cm}^{2}$ to $5 \times 10^{15}$ ions per $\mathrm{cm}^{2}$. The experimental results revealed a high degree of diamond structural damage after the ion implantation process reaching up to $69 \%$ of disordered atoms in the samples. The prepared Er-doped diamond samples annealed at the temperatures of 400,600 and $800{ }^{\circ} \mathrm{C}$ in a vacuum revealed clear luminescence, where the $\langle 110\rangle$ cut sample has approximately 6-7 times higher luminescence intensity than the $\langle 001\rangle$ cut sample with the same ion implantation fluence. The reported results are the first demonstration of the Er luminescence in the single crystal diamond structure for the near-infrared spectral region.
\end{abstract}

\section{Introduction}

Diamond exhibits outstanding physical and chemical properties such as extremely high chemical and thermal resistance, and the highest hardness and thermal conductivity among all materials.

\footnotetext{
${ }^{a}$ Department of Inorganic Chemistry, University of Chemistry and Technology, Technická 5, 16628 Prague, Czech Republic. E-mail: cajzlj@vscht.cz

${ }^{b}$ Institute of Photonics and Electronics, Czech Academy of Sciences, v.v.i., Chaberská 57, 18251 Prague, Czech Republic

${ }^{c}$ Nuclear Physics Institute, Czech Academy of Sciences, v.v.i., 25068 Řež, Czech Republic

${ }^{d}$ Department of Physics, J.E. Purkinje University, České mládeže 8 , 40096 Ústí nad Labem, Czech Republic

${ }^{e}$ Institute of Physics, Czech Academy of Sciences, v.v.i., Cukrovarnická 10/112, 16200 Prague, Czech Republic

${ }^{f}$ Helmholtz-Zentrum Dresden-Rossendorf, Institute of Ion Beam Physics and Materials Research, Bautzner Landstraße 400, 01328 Dresden, Germany

$\dagger$ Electronic supplementary information (ESI) available. See DOI: 10.1039/c6cp08851a
}

Moreover, diamond is also biocompatible due to its carbon nature. ${ }^{1}$ Thanks to these properties, diamond is intensively studied for use in electronics, photonics and integrated quantum photonics. In electronics, diamond is mostly studied for its use as p- and n-type semiconductors. For these purposes, it has been doped with various dopants to create a p-type diamond (doping with boron $^{2,3}$ ) or n-type diamond (doping with nitrogen, phosphorus or sulphur $\left.{ }^{4}\right)$.

In the photonics field, micro- or nanocrystalline diamond (MCD or NCD) thin films grown on silicon or $\mathrm{SiO}_{2}$ have been used for special High Index Contrast (HIC) waveguides. ${ }^{5}$ The estimated relative refractive index difference $\Delta n$ reaches a high value of about $30 \%$ for the glass/NCD. This high value of refractive index difference makes diamond HIC waveguides suitable for photonic waveguiding structures with a high density of integration. ${ }^{6,7}$ However, the high degree of optical attenuation mainly due to the scattering of light on grain boundaries could limit the real applications of NCD waveguides. ${ }^{8}$ 
Optically active impurities in diamond are called colour centres. Nowadays, colour centres in diamond are attracting growing attention owing to their highly promising application in quantum information processing where they can serve as ultra bright, highly polarized, and narrow-band single-photon emitters. ${ }^{9}$ The highly explored field of integrated quantum photonics makes use of nitrogen-vacancy (NV) centres in diamond; the most important among them are negatively charged nitrogen-vacancy $\left(\mathrm{NV}^{-}\right)$ centres. The NVs in diamond are used due to their spin properties as quantum bits (also called 'qubits') - NV centres can store, transfer and process qubits at room temperature. ${ }^{10-12}$ Besides nitrogen-vacancy centres in diamond, other colour centres created by doping with $\mathrm{Si}^{13}{ }^{\mathrm{Ni}}{ }^{14} \mathrm{Cr}^{15}$ or $\mathrm{C}^{16}$ have been identified as diamond-based single-photon emitters. All the structural defects mentioned above exhibit emission at wavelengths shorter than approx. $800 \mathrm{~nm}$. The photophysical properties of most colour centre defects have been summarized in ref. 17 . Concerning the near infrared region of about 900-2000 nm only a few non-defined colour centres exist. Some of the centres were created by diamond single crystal annealing ${ }^{18}$ or by CVD with nanoparticles of diamond and Ni. ${ }^{19}$ Recently the effect of the diamond microstructure on the photophysical properties of colour centres has also been studied in detail. ${ }^{20,21}$

Lanthanides are vital elements in lighting and imaging technologies and future quantum memory applications owing to their narrow spectral energy transitions. Diamond doping with lanthanides has been published rarely so far. ${ }^{22}$ However, the theoretical and experimental study of Eu-doping into diamond thin films using electrostatically assembled europium(III) chelate molecules combined with the CVD technique gave evidence that the successful diamond doping with lanthanides is possible. ${ }^{23}$ In that study the luminescence of Eu had the maximum at about $620 \mathrm{~nm}$.

Erbium is one of the most frequently used elements in photonics due to its strong narrow emission in the third telecommunication window. To our knowledge erbium doping of bulk diamond has not been experimentally done yet. The doping of diamond with erbium ions could be the solution for the compensation of optical losses in NCD waveguides in the NIR spectral region around $1.5 \mu \mathrm{m}$.

Electron density is the key quantity determining the total and cohesive energies, the crystal field potentials, interatomic forces, and a number of related physical and chemical quantities. The DFT (Density Functional Theory) approach based on quantum mechanical calculations of electron density is one of the most frequently used techniques for the simulation of solid state structures. Moreover, the electronic and magnetic properties of materials can be calculated using this approach. In the last decade the theoretical modeling studies of halogens, ${ }^{24}$ transition metals, ${ }^{25}$ boron $^{26}$ or oxygen, ${ }^{27}$ in diamond have been performed using DFT. Usually the optimized structures as well as the electronic band structures and defect forming energies have been reported and discussed. For the simulation of europium in diamond, it has been found that the most stable configuration is the six-fold coordination shell of europium with single carbon vacancy. ${ }^{23}$

Our study focuses on the doping of the diamond structure with erbium ions. The theoretical study of erbium positions in the Er:diamond single-crystal structure with and without vacancies was done. The doping of diamond with erbium ions was done by the ion implantation technique. Ion implantation is a well-known doping technique with one of the highest versatilities of the preparation conditions for the creation of colour centres in diamond. This is because the ion implantation makes it easy to control the depth of the implanted region and the concentration of the implanted ions. However, depending on the applied ion implantation energy and fluence, the method can cause high material damage to targets. Nevertheless, there are some possibilities to recover the diamond structure by post-implantation annealing. ${ }^{28}$ In our experiments various fluences of erbium ions were thus used to compare the different degree of structural damage caused by ion implantation. The experimental results were combined with the theoretical study.

\section{Experimental}

\subsection{DFT simulations}

Investigation of the structural properties of erbium in the single-crystal diamond structure as well as identification of the most favourable positions of erbium in the diamond structure was done using a theoretical structural modelling approach based on a Density Functional Theory (DFT) ab initio calculation method (generalized gradient approximation) with Vanderbilttype ultrasoft pseudopotentials. The calculations were carried out on crystal supercells using periodic boundary conditions for the crystal structures. The CASTEP ${ }^{29}$ package was used to perform structure geometry optimizations and to obtain the accurate values of the total energies of the respective supercell structural models. The results were obtained as static $(0 \mathrm{~K})$ and atomic positions are relaxed according to the calculated forces on each atom.

We explored two different Er-doped diamond structural models and performed geometry optimizations with the calculations of total energies of the pertinent structures. Moreover we performed additional calculations by introducing 1 to 4 carbon vacancies incorporated in the vicinity of the erbium atom. The initial structural models - taken as the input for the geometry optimization calculations - differed in Er positions within the structure for the determination of the most favourable structure arrangement. Fig. 1 depicts the investigated structural models of Er:diamond with erbium in (a) the interstitial position of a tetrahedral void and (b) the substitutional position of carbon. Geometry optimization calculations were done with $3 \times 3 \times 3$ supercell models (based on the non-primitive diamond cubic unit cell consisting of 8 atoms), yielding the total number of atoms of 216 for the parent undoped diamond. In the Er:diamond models with various numbers of vacancies, the same $3 \times 3 \times 3$ diamond supercell base was used, where one atom of erbium was involved per total number of 212 to 217 atoms for the respective models. The size of the main unit $(3 \times 3 \times 3)$ was chosen according to the most commonly used experimental bulk concentration of erbium, which is about 0.5 at\%, i.e. for 217 atoms (model depicted in Fig. 1a), and we obtained an erbium concentration of:

$$
\frac{1 \mathrm{Er}}{217 \text { atoms }} \cdot 100 \%=0.46 \mathrm{at} \%
$$


(a)

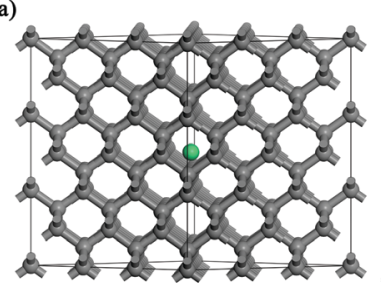

g. 1 The structural models of Er:diamond with the erbium ion (a) in the interstitial position of a tetrahedral void and (b) in the substitutional position of carbon. The structural models were $3 \times 3 \times 3$ supercells.

accordingly for other structural models. Additionally the calculations with supercells introducing 1 to 4 carbon vacancies in the vicinity of the erbium atom were done. For the calculations with vacancies, only the carbon vacancies in the 1st layer of atoms surrounding the erbium atom were chosen. The nearest Er vicinity for vacancies was chosen due to the fact that the first layer of atoms is most compensating the introduced Er defect. Here it is important to note that the carbon atoms closest to the Er atom are different for the substitutional and the interstitial position, i.e. the carbon atoms chosen for the vacancies in the vicinity of Er are different for the substitutional and the interstitial Er position. Mostly there is the difference in the distances between the vacancy carbon atoms for both models.

The calculations were spin-polarized using ultrasoft pseudopotentials, the BFGS (Broyden-Fletcher-Goldfarb-Shanno) algorithm for geometry optimizations, and the exchange functional type GGA-RPBE (Generalized Gradient Approximation-Revised Perdew-Burke-Ernzerhof) (revised with corrections for dispersion effects). The GGA-RPBE exchange functional was chosen according to the results of our test calculations with the parent diamond structure and comparison of the calculated cohesive energies with the experimental value. The following functionals were investigated and compared: PW91 (Perdew-Wang 91 (1991)), PBE (PerdewBurke-Ernzerhof (1996)), RPBE, PBE-sol (Perdew-Burke-Ernzerhof for solids (2008)), and WC (Wu-Cohen (2006)).

The energy cutoffs and $k$-point mesh densities were chosen according to the convergence tests for the appropriate settings for accurate results to be found. The plane-wave cutoff energy was set to $360 \mathrm{eV}$ (CASTEP 'Ultrafine' setting) and the $k$-point set of the Monkhorst-Pack grid $1 \times 1 \times 1$ (one irreducible $k$-point) corresponding to the $k$-spacing of $0.0935 \AA^{-1}$ (the used supercells were very large in real space, so that the Brillouin zone was small and no $k$-point sampling was therefore required). Geometry optimizations were performed with the convergence criteria of an energy change of $10^{-5} \mathrm{eV}$ per atom, a maximum force of $3 \times 10^{-2} \mathrm{eV}^{-1}$, a maximum stress of $5 \times 10^{-2} \mathrm{GPa}$ and a maximum atom displacement of $10^{-3} \AA$.

Charge analysis in the calculations was done using three analysis methods: charge equilibration (QEq), Mulliken and Hirshfeld. The QEq method is based on the equilibration of atomic electrostatic potentials with respect to a local charge distribution. $^{30}$ Mulliken charges and bond populations are calculated according to the formalism described by Segall et al. ${ }^{31,32}$ Population analysis of the resulting projected states was performed using the Mulliken formalism. ${ }^{33}$ Hirshfeld charges ${ }^{34}$ are defined relative to the deformation density representing a difference between the molecular and unrelaxed atomic charge densities.

\subsection{Doping of diamond with erbium}

The Er:diamond samples were prepared from single-crystal diamond wafers with the $Z\langle 001\rangle$ and $X\langle 110\rangle$ crystallographic orientations (fabricated by Element Six Technologies Ltd, UK). The dimensions of the samples were $3 \times 3 \times 0.3 \mathrm{~mm}$. Thoroughly pre-cleaned wafers were implanted with $190 \mathrm{keV}$ energy $\mathrm{Er}^{+}$ions ( $7^{\circ}$ off-axis to avoid channeling) with fluences of $1.0 \times 10^{14}, 1.0 \times$ $10^{15}$ and $5.0 \times 10^{15}$ ions per $\mathrm{cm}^{2}$. The ion implantation was performed on a $200 \mathrm{kV}$ ion implanter in the Helmholtz-Zentrum, Dresden-Rossendorf, Germany. The beam was scanned through an aperture of typical dimensions $1 \times 1 \mathrm{~cm}^{2}$, resulting in a beam current density of typically $0.25 \mu \mathrm{A}$ per $\mathrm{cm}^{2}$ on the target. The prepared Er:diamond samples were annealed after the ion implantation in a vacuum $\left(10^{-4} \mathrm{mbar}\right)$ for 1 hour at 3 different temperatures $\left(400,600\right.$ and $\left.800{ }^{\circ} \mathrm{C}\right)$.

\subsection{Materials characterization}

The prepared samples of Er:diamond were characterized primarily in terms of the structural changes connected with the erbium incorporation in the parent diamond structure by the ion implantation process. The erbium concentration depth profiles were determined by Rutherford Backscattering Spectroscopy (RBS). The analysis was performed on a Tandetron $4130 \mathrm{MC}$ accelerator using a $2.0 \mathrm{MeV} \mathrm{He}^{+}$ion beam. The collected data were evaluated and transformed into concentration-depth profiles using GISA $3.99^{35}$ and SIMNRA $6.06^{36}$ codes, utilizing the crosssectional data from IBANDL. ${ }^{37}$ Using the experimental set-up of RBS analysis, it was possible to achieve the typical depth resolution better than $10 \mathrm{~nm}$ and the lowest detected concentration of Er was about 0.005 at $\%$. The structural modification of the single crystal diamond structure after the ion implantation process was examined by the RBS/channeling measurement using a $2 \mathrm{MeV} \mathrm{He}^{+}$ion beam.

The Raman spectra of the prepared samples were acquired using a DXR Raman Microscope spectrometer (Thermo Scientific) equipped with a confocal Olympus microscope. The excitation source used was a solid-state Nd:YAG laser (wavelength $532 \mathrm{~nm}$, maximum power $10 \mathrm{~mW}$ ). Measurement conditions were: $2-8 \mathrm{~mW}$ power, 10 accumulations of 10 second scans, grating with 900 lines per $\mathrm{mm}$ and aperture of $25 \mu \mathrm{m}$ pinhole. As detector a multichannel thermoelectrically cooled CCD camera was used. 50-times magnification provided a measurement spot-size of $\sim 1 \mu \mathrm{m}^{2}$.

The photoluminescence spectra of investigated samples were collected in the range of 1440-1650 $\mathrm{nm}$ at room temperature. A semiconductor laser POL 4300 emitting at $980 \mathrm{~nm}$ was used for the excitation. The luminescence radiation was detected using a two-step-cooled Ge detector J16 (Teledyne Judson Technologies). To scoop specific wavelengths, a double monochromator SDL-1 (LOMO) was used. 


\section{Results}

\subsection{The theoretical structural modelling of Er:diamond}

3.1.1 Undoped diamond. The calculated cohesive energies of the parent diamond using different exchange functionals (keeping all the calculation parameters the same except for the exchange functional used) are summarized in Table 1 . The cohesive energy of the diamond using the RPBE exchange functional ( $7.50 \mathrm{eV}$ per atom) is in an excellent agreement with the experimental value of $7.58 \mathrm{eV}$ per atom (being approx. 1.0\% lower). Hence, the used GGA-RPBE functional calculation slightly underestimates the cohesive energy of diamond. The cohesive energy is also in an excellent agreement with the values previously reported using the complex Quantum Monte Carlo study (7.503 eV per atom). ${ }^{38}$ One can argue at this point that the comparison of exchange functionals for the final models with the incorporated erbium atom can differ from these initial calculations performed on pure diamond. However, this approach of choosing the exchange functional according to the calculation of energy of the undoped diamond is possible thanks to the fact that the vast majority of atoms in supercells $(3 \times 3 \times 3)$ used in calculations are the carbon atoms - i.e. from 211 to 216 carbon atoms and 1 erbium atom - therefore there will also be a majority of carbon-carbon bonds and just very few carbon-erbium bonds (the bonds can be of different chemical and physical nature including dispersive forces).

3.1.2 Cohesive and defect formation energies - Er-doped diamond. The following approach was applied in our study: first, the geometry optimization calculations were performed and it was investigated whether (and which) structural supercell models converged; second it was determined how the geometry around the central erbium ion changed after the geometry optimization; third, the cohesive energies of the structures were calculated together with the calculation of defect formation energies for the incorporation of erbium atom defects; finally, the cohesive energies as well as defect formation energies for the different structural supercell models were compared in order to estimate their relative stability (i.e. the higher cohesive energy means the higher stability of the structure) and the possibility to incorporate erbium defects into the structure of diamond (evaluated by the lowest formation energy).

Cohesive energies were calculated according to eqn (2), ${ }^{38,40}$ where $E_{\text {Cohesive }}$ is the cohesive energy per atom of the simulated

Table 1 The calculated cohesive energies of the parent undoped diamond using various exchange functionals. The deviation from the diamond experimental cohesive energy is also noted

\begin{tabular}{llc}
\hline & $\begin{array}{l}\text { Calculated cohesive } \\
\text { energy } E_{\text {Cohesive }} \\
\text { Structure }\end{array}$ & $\begin{array}{l}\text { Relative change } \\
\text { from experimental } \\
E_{\text {Cohesive }}[\%]\end{array}$ \\
\hline $\begin{array}{llc}\text { Diamond } \\
\text { (Experimental value) }\end{array}$ & 7.58 & 0.0 \\
Diamond (PW91) & 7.92 & 4.5 \\
Diamond (PBE) & 7.95 & 4.8 \\
Diamond (RPBE) & 7.50 & $-\mathbf{1 . 0}$ \\
Diamond (PBE-sol) & 8.62 & 13.7 \\
Diamond (WC) & 8.41 & 11.0
\end{tabular}

structure, $n$ is the number of all atoms in the calculated structure, $n_{i}$ is the number of atoms of one type (e.g. in our case, $i$ was 1 for carbon and 2 for erbium element types), $E_{\mathrm{at}, i}$ is similarly the atomic energy of an atom of one type (e.g. $E_{\mathrm{at}, \mathrm{C}}$ and $E_{\mathrm{at}, \mathrm{Er}}$ in our case), $k$ is the number of atom types, and $E_{\text {Total }}$ is the calculated total energy of the structure after the geometry optimization.

$$
E_{\text {Cohesive }}=\frac{\sum_{i=1}^{k}\left(n_{i} \cdot E_{\mathrm{at}, i}\right)-E_{\mathrm{Total}}}{n}[\mathrm{eV} \text { per atom }]
$$

In order to evaluate the cohesive energies of the structures, it was necessary to calculate the total energies of the corresponding isolated atoms in the structures (using the same exchange functionals and calculation quality settings). For the calculation of the carbon atom, we used a $10 \times 10 \times 10 \AA$ cube with a single carbon atom in the centre giving enough space around the atom for it to be considered as an isolated atom. For the erbium atom, we used a slightly larger cube of $15 \times 15 \times 15 \AA$ to ensure the isolation of an atom as well. The total energies of $-147.501 \mathrm{eV}$ and $-5225.094 \mathrm{eV}$ were obtained for the isolated carbon and erbium atom, respectively.

Defect formation energies for the neutral Er defects in diamond supercell models were calculated according to eqn (3), ${ }^{41,42}$ where $E_{\text {Total }}\left[\mathrm{X}^{0}\right]$ is the total energy of the supercell with the neutral defect $\mathrm{X}, E_{\text {Total }}[\mathrm{bulk}]$ is the total energy of the bulk parent supercell, $n_{i}$ are the number of atoms of one type (like in eqn (1)) that have been removed from or added to the structure in order to create a defective supercell $\left(n_{i}\right.$ is positive for added atoms and negative for removed atoms), and $\mu_{i}$ are the chemical potentials of the corresponding removed or added atoms.

$$
E_{\mathrm{f}}\left[\mathrm{X}^{0}\right]=E_{\text {Total }}\left[\mathrm{X}^{0}\right]-\left\{E_{\text {Total }}[\text { bulk }]+\sum_{i=1}^{k}\left(n_{i} \cdot \mu_{i}\right)\right\}[\mathrm{eV}]
$$

For the selected Er:diamond supercell models the defect formation energies of the charged Er defects were also calculated. The equation for the calculation of defect formation energies of the charged defects in crystal supercell models was obtained by adding 2 extra elements in the equation for neutral defects (see eqn (3)) that account for the charge incorporation in the defective supercell. The calculation of defect formation energies of charged Er defects in diamond was performed according to eqn (4), ${ }^{40-42}$ where $E_{\text {Total }}\left[\mathrm{X}^{q}\right]$ is the total energy of the supercell with the charged defect $\mathrm{X}, E_{\text {Total }}[\mathrm{bulk}], n_{i}$ and $\mu_{i}$ are the same as in eqn (3), $q$ is the charge of the defect, $\Delta \mu_{\mathrm{e}}$ is the shift of the Fermi energy level with respect to the parent bulk valence band maximum $E_{\mathrm{VBM}}, \Delta V$ is the term used for the alignment of the reference potential of the bulk and defect supercell, and $E_{\text {corr }}^{q}$ is the finite-size supercell correction. In our case we were not analysing the effects of $\Delta V$ and $E_{\text {corr }}^{q}$ because of the very low concentration of Er defects ( 1 atom of Er in the supercell) and the relatively large dimensions of the supercell. Sometimes the term $q\left(E_{\mathrm{VBM}}+\Delta \mu_{\mathrm{e}}\right)$ is being defined as the $q\left(\mu_{\mathrm{e}}\right)$ which is the Fermi level $\mu_{\mathrm{e}}$ multiplied by the charge $q$, i.e. the thermodynamic work required to add one electron to the body (Fermi level $\mu_{\mathrm{e}}$ ) multiplied by the charge $q$ (which refers to the number 
of electrons added or removed). Since the calculations are done at static positions $(0 \mathrm{~K})$, the Fermi level equals Fermi energy. In the calculations the chemical potential for carbon $\mu_{\mathrm{C}}$ was taken from the calculated energy of the undoped diamond supercell as the supercell energy divided by the number of atoms in the supercell. The chemical potential of erbium $\mu_{\mathrm{Er}}$ was calculated from the total energy of metallic erbium (with a hexagonal structure, space group no. 194) divided by the number of erbium atoms.

$$
\begin{aligned}
E_{\mathrm{f}}\left[\mathrm{X}^{q}\right]= & E_{\text {Total }}\left[\mathrm{X}^{q}\right] \\
& -\left\{E_{\text {Total }}[\text { bulk }]+\sum_{i=1}^{k}\left(n_{i} \cdot \mu_{i}\right)-q\left(E_{\mathrm{VBM}}+\Delta \mu_{\mathrm{e}}+\Delta V\right)\right\} \\
& +E_{\text {corr }}^{q}[\mathrm{eV}]
\end{aligned}
$$

The calculated cohesive energies of the Er:diamond structures with various numbers of carbon vacancies (in the vicinity of $\mathrm{Er}$ atom) together with the calculated defect formation energies are summarized in Table 2 - here $\mathrm{Er}_{\text {int }}$ is the erbium in the interstitial position and $\mathrm{Er}_{\text {sub }}$ is the erbium in the substitutional position of carbon. The models with erbium in the interstitial position of the tetrahedral void had overall lower cohesive energies and higher defect formation energies in comparison with erbium in the substitutional carbon position, making this position less favourable for erbium defects. The highest calculated cohesive energies and lowest defect formation energies were obtained for models with erbium in the substitutional carbon position with 1 or 3 vacancies (in the vicinity of the erbium atom).

The defect formation energies of charged Er-defects for the most favourable Er:diamond supercells are summarized in Table 3. For the calculation of different charged states only the most probable values of charge states of erbium in solids were chosen, i.e. 0 (neutral), $+1,+2$ and +3 . From the results it turned out that for the given Er concentration (approx. 0.5 at\%) the defect formation energies decrease with the higher charge state of Er.

3.1.3 Geometry, band structure and charge analysis - Er-doped diamond. Fig. 2 depicts the models of Er:diamond with erbium in (a) the interstitial tetrahedral position and (b) the substitutional carbon position after performing the geometry optimization.
Considering the changes in the vicinity of the erbium atom in both models, the carbon atoms that were closest to the Er atom (in the $\mathrm{Er}_{\text {int }}$ model the four carbon atoms surrounding the tetrahedral interstitial position; in the $\mathrm{Er}_{\text {sub }}$ model the four carbon atoms forming a bond to the substituted carbon atom) were displaced away from the Er atom by about $22 \%$ and $29 \%$ based on the initial Er-C distance for the $\mathrm{Er}_{\text {int }}$ and $\mathrm{Er}_{\text {sub }}$ models, respectively. The $\mathrm{C}-\mathrm{C}$ bond lengths of the closest atoms became about $17 \%$ larger for the $\mathrm{Er}_{\text {int }}$ model and about 4\% shorter for the $\mathrm{Er}_{\text {sub }}$ model. It is obvious from the images (Fig. 2) that the surroundings of the central erbium atoms (about 1st and 2nd layers of the atoms) become larger and more 'inflated', but in the 3rd, 4th and 5th layers the carbon atoms move negligibly.

Concerning the geometry optimization of the models of Er:diamond in both interstitial and substitutional positions with up to four vacancies in the vicinity of the Er atom, the calculations showed interesting phenomena. When the Er atom was in the interstitial tetrahedral position with one or more vacancies in the Er atom vicinity, the Er atom moved in the direction of - or almost completely to - the substitutional carbon vacancy position. This was observed despite the fact that the radii of the carbon vacancy $\left(\mathrm{V}_{\text {carbon }}\right)$ and the tetrahedral diamond vacancy $\left(\mathrm{V}_{\text {tetrahedral }}\right)$ are similar in diameter - with the diamond tetrahedral vacancy being just approximately $1 \mathrm{pm}$ larger than carbon in tetrahedral coordination (see Table 4). After the geometry optimization, the Er atom was almost entirely in the substitutional carbon position. Here it is worth mentioning that the relaxed structure of $\mathrm{Er}$ in the interstitial position with one vacancy almost equals the structure with $\mathrm{Er}$ in the substitutional position with no vacancy (if the convergence criteria are very strict, the calculation time would be very long and the two structures would equal each other completely). However, the relaxed interstitial structures with more than one vacancy are no longer matching the substitutional structures because of the difference in the vacancy carbon positions for the substitutional and the interstitial positions. So from this analysis it turned out that Er is willing to occupy the substitutional position rather than the interstitial one despite the almost identical size of $\mathrm{V}_{\text {tetrahedral }}$ and $\mathrm{V}_{\text {carbon }}$.

For the supercell models with the highest cohesive energies (lowest defect formation energies) - i.e. erbium in the substitutional carbon position with 0 to 4 vacancies - the nearest neighbours of

\begin{tabular}{|c|c|c|c|}
\hline Structure & $\begin{array}{l}\text { Calculated cohesive } \\
\text { energy [eV per atom] }\end{array}$ & $\begin{array}{l}\text { Relative energy change from } \\
\text { experimental } E_{\text {Cohesive }}[\%]\end{array}$ & $\begin{array}{l}\text { Defect formation } \\
\text { energy }[\mathrm{eV}]\end{array}$ \\
\hline Diamond (Experimental value) ${ }^{39}$ & 7.58 & 0.0 & - \\
\hline $\mathrm{Er}_{\text {int }}:$ diamond (0 vacancies) & 7.340 & -3.2 & 30.14 \\
\hline $\mathrm{Er}_{\text {int }}$ :diamond (2 vacancies) & 7.333 & -3.3 & 16.71 \\
\hline $\mathrm{Er}_{\text {int }}$ :diamond (3 vacancies) & 7.290 & -3.8 & 18.39 \\
\hline $\mathrm{Er}_{\text {int }}$ :diamond (4 vacancies) & 7.234 & -4.6 & 23.00 \\
\hline $\mathrm{Er}_{\text {sub }}:$ diamond (1 vacancy) & 7.425 & -2.0 & 11.40 \\
\hline $\mathrm{Er}_{\text {sub }}$ :diamond (2 vacancies) & 7.421 & -2.1 & 12.22 \\
\hline $\mathrm{Er}_{\text {sub }}$ :diamond (3 vacancies) & 7.428 & -2.0 & 10.74 \\
\hline $\mathrm{Er}_{\text {sub }}$ :diamond (4 vacancies) & 7.407 & -2.3 & 15.02 \\
\hline
\end{tabular}

Table 2 The calculated cohesive energies of the diamond and Er:diamond structures including various numbers of vacancies together with the calculated defect formation energies 
Table 3 The calculated defect formation energies of charged Er-defects in the substitutional position in the diamond structure including various numbers of vacancies in the vicinity of erbium

\begin{tabular}{|c|c|c|}
\hline & One vacancy & Three vacancies \\
\hline Charge & Defect formation energy $[\mathrm{eV}]$ & Defect formation energy $[\mathrm{eV}]$ \\
\hline 0 & 11.40 & 10.74 \\
\hline+1 & 6.764 & 7.678 \\
\hline+2 & 3.815 & 4.306 \\
\hline+3 & 0.983 & 1.263 \\
\hline
\end{tabular}

(a)

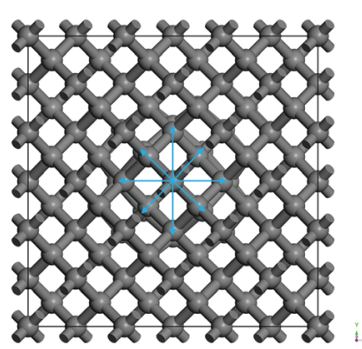

(b)

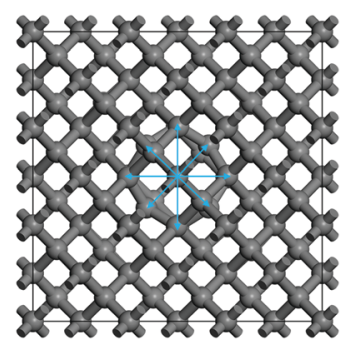

Fig. 2 The models of the Er:diamond structure after the geometry optimization for the erbium ion in (a) interstitial tetrahedral position and (b) substitutional carbon position. The structure images are $X Y$ view perpendicular to the $Z$-axis. In the pictures up to 4 carbon neighbouring layers can be seen in the vicinity of the $\mathrm{Er}$ atom.

Table 4 The radii of the $\mathrm{Er}^{3+}$ ion, the carbon atom (i.e. also carbon vacancy) and the tetrahedral diamond vacancy

\begin{tabular}{ll}
\hline Atom/ion/vacancy & Radius [pm] \\
\hline $\mathrm{Er}^{3+}$ (VI coordination) & $89.0^{44}$ \\
$\mathrm{C}$ (tetrahedral coordination) $=\mathrm{V}_{\text {carbon }}$ & $76.7^{45,46}$ \\
$77.7^{a}$ \\
$\mathrm{~V}_{\text {tetrahedral }}$ \\
a Calculated as the half-distance between the center of the tetrahedral \\
vacancy and the center of the nearest neighbouring carbon atom in the \\
diamond structure.
\end{tabular}

the Er atom were investigated. The results are summarized in Table 5 with the notion of the coordination number, structure type and bond lengths. From the results it can be seen that the higher number of vacancies in the vicinity of erbium is causing a higher coordination number of erbium, i.e. for 0 to 4 vacancies the coordination numbers were from 4 up to 12 , respectively. The average Er-C bond lengths were approx. 200 to $260 \mathrm{pm}$. The structure images for the two most favourable structural models are shown in Fig. 3 for ( $a$ and b) one carbon vacancy and (c and d) three carbon vacancies, respectively. The Er coordination in the supercell model with one carbon vacancy was found to be octahedral (coordination 6) with the bond lengths of around $213 \mathrm{pm}$. For the supercell model with three vacancies the Er coordination was 10 . The structure of the coordination $\mathrm{X}$ exhibited a trigonal symmetry and is as follows: around the Er atom was a hexagonal ring with the longest bonds of $262 \mathrm{pm}$, under (above) the ring were three atoms in trigonal arrangement with the bond lengths of around $228 \mathrm{pm}$ and above (under) the ring was one atom with the shortest bond length of $222 \mathrm{pm}$. The coordination number 10 for erbium was already published in the literature, e.g. the $\mathrm{Er}^{\mathrm{III}}$-erythritol complex ${ }^{47}$ which has an irregular $\mathrm{ErO}_{10}$ coordination geometry where the $\mathrm{Er}^{3+}$ cation is chelated by three nitrate anions (forming a hexagonal circle or ring), one erythritol molecule (forming a trigonal arrangement of O-atoms under/above the hexagonal ring), and one ethanol molecule (being a single top under/above the hexagonal ring). Other similar Er complexes with coordination 10 were published as well in e.g. ref. 48 and 49.

The band structure can provide useful information to explain some physical properties of a material, so its theoretical calculation is important. For a parent diamond structure and the most stable Er:diamond structure (with Er in the substitutional position with 3 vacancies in the vicinity) the calculations of the partial density of states (PDOS) for all the orbitals (s, p, d, and f) as well as the band structure analysis (including the band gap energy) were performed. The results are depicted in Fig. 4. It can be seen from the graphs that the total density of states (DOS) of the parent diamond only consists of $\mathrm{s}$ and $\mathrm{p}$ states of the carbon atoms. In the second case of the Er:diamond structure also the f orbitals belonging to the incorporated erbium atom can be seen near the Fermi level.

Charge analysis was done using three charge analysis formalisms: Mulliken, Hirshfeld and QEq (for a description see Section 2.1). The calculations were done for the Er-defect in the substitutional position in the diamond structure with various numbers of vacancies and are summarized in Table 6 . The charge values are stated in the elementary charge $e$ (approx. $1.6021766208 \times$ $\left.10^{-19} \mathrm{C}^{50}\right)$. In the Mulliken charge analysis the trend is that with the increasing number of vacancies the calculated charge decreases from about $+3.7 e$ to $+2.58 e$. In the Hirshfeld charge analysis the charge was around $+0.3 e$, having the lowest value for the 2 vacancies of $+0.29 e$, the highest value for the 3 vacancies of $+0.39 e$, and for 0,1 , and 4 vacancies a value of $+0.32 e$. The difference is very high between the two approaches. If one considers the charge neutrality condition for the resulting Er-doped diamond, the carbon atoms in the vicinity of the Er atom with the charge of

Table 5 The nearest neighbouring orderings of the erbium atom in the substitutional position in the diamond structure supercells after the geometry optimization and the resulting coordination structures of erbium with carbon atoms

\begin{tabular}{llll}
\hline $\begin{array}{l}\text { Number of } \\
\text { vacancies }\end{array}$ & $\begin{array}{l}\text { Coordination number } \\
\text { (bonding) }\end{array}$ & Structure type & Bond lengths \\
\hline 0 & $4\left(\mathrm{ML}_{4}\right)$ & Tetrahedron & $4 \times 199 \mathrm{pm}$ \\
1 & $6\left(\mathrm{ML}_{6}\right)$ & Octahedron & $6 \times 213 \mathrm{pm}$ \\
2 & $6\left(\mathrm{ML}_{6}\right)$ & Trigonal prism & $6 \times 222-224 \mathrm{pm}$ \\
3 & $10\left(\mathrm{ML}_{10}\right)$ & "Trigonal structure with hexagonal ring" & $1 \times 222 \mathrm{pm}, 3 \times 228 \mathrm{pm}, 6 \times 260-264 \mathrm{pm}$ \\
4 & $12\left(\mathrm{ML}_{12}\right)$ & Cuboctahedron & $12 \times 260-263 \mathrm{pm}$
\end{tabular}


(a)

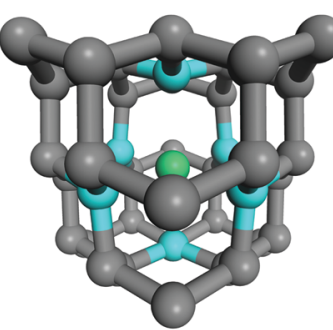

(c)

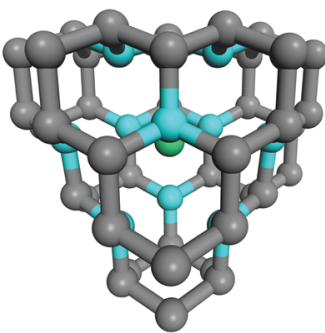

(b)

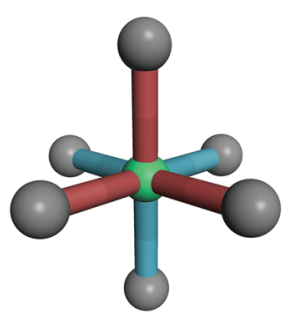

(d)

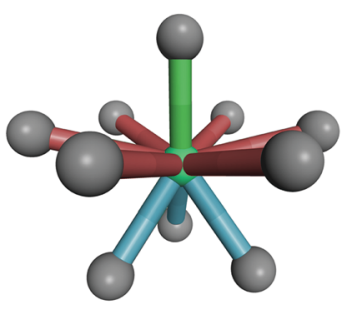

Fig. 3 The nearest neighbours of the erbium atom in the substitutional position in the diamond structure supercells after the geometry optimization and the resulting coordination structures of erbium with carbon atoms, for ( $a$ and b) one carbon vacancy and (c and d) three carbon vacancies, respectively. Atoms highlighted with cyan colour in the Er neighbouring clusters ( $a$ and $c$ ) show the nearest carbon atoms to erbium that are forming the coordination structures ( $b$ and $d$ ). In the coordination structure images ( $b$ and $d$ ) the same bond colours represent the same bond lengths; (b) red $=213.1 \mathrm{pm}$ and blue $=212.9 \mathrm{pm}$, and (d) red $=261.8 \mathrm{pm}$, blue $=$ $227.7 \mathrm{pm}$, and green $=221.8 \mathrm{pm}$.

around +3 from Mulliken analysis would have the total charge of approx. -3 which is a fairly high value for a covalently bonded structure like diamond with all carbon atoms having zero charge. Therefore the value of around +0.3 to +0.4 from Hirshfeld analysis seems to be more reasonable for the real system with the Er defect. The charge equilibration method gave the charge values of around $+0.88 e$ and almost did not change for the different models with various numbers of vacancies. This is probably caused by the fact that the charge equilibration method is mainly used for the molecules and with the very large systems it could not account for the small changes in the vicinity of one atom.

\subsection{The experimental results of Er:diamond}

Three samples of Er-doped single-crystal diamond were prepared and analysed. An overview specifying the conditions of ion implantation is provided in Table 7. For the erbium concentration depth profile determination, the RBS method was used. In Fig. 5a the erbium concentration depth profiles are depicted for the Er:diamond samples prepared by using the ion implantation fluences of $1.0 \times 10^{14}$ ions per $\mathrm{cm}^{2}, 1.0 \times 10^{15}$ ions per $\mathrm{cm}^{2}$ and $5.0 \times 10^{15}$ ions per $\mathrm{cm}^{2}$. The ion projected range $R_{\mathrm{p}}$ was derived as the mean value of erbium depth profiles determined by RBS and was supposed to obey a Gaussian distribution, while $\Delta R_{\mathrm{p}}$ was determined by evaluating the profile standard deviation. These values were extracted from the experimental Er-concentration depth profiles and were compared with the predictions given by SRIM simulations, which are summarized in Table 7. The relative concentrations of the disordered atoms $N_{\mathrm{D}} / N$ were calculated from the area of the deeper-lying disordered peak

(see Fig. 6) using the well-known procedure described in ref. 43. The parameters $N_{\mathrm{D}} / N$ were deduced from eqn (5), including the region broader than the surface peak to cover the whole $\mathrm{Er}$ implanted layer. The damage accumulation is mainly pronounced in the subsurface region at a channel of about 200 (see Fig. 6) in the aligned spectra (the aligned spectrum is obtained by recording back-scattered ions under the condition that the ion beam is aligned along the main axis of the crystallographic orientation). Thus the average relative disorder density in the estimated layer is lower than that in the subsurface layer where the almost fully disordered structure appeared.

$$
N_{\mathrm{D}} / N=\frac{\chi_{\mathrm{D}}-\chi_{\mathrm{V}}}{1-\chi_{\mathrm{V}}}
$$

In eqn (5) $\chi_{\mathrm{V}}$ is the minimum yield in the aligned parent diamond spectrum and $\chi_{\mathrm{D}}$ is the minimum yield in the aligned spectra of the implanted samples. The minimum yields are determined as the relative numbers of de-channelled ions collected in the spectrum aligned along the crystallographic axis in the region of interest appropriate for the examined element (e.g. carbon or erbium in this case) and the details can be found in ref. 51 .

As expected, the erbium concentrations in the surface layers were increasing according to the increasing ion implantation fluences used. The highest erbium concentrations in the samples of about 0.045 at $\%, 0.225$ at $\%$ and 0.800 at $\%$ were attained, respectively, for the increasing ion implantation fluences $(1.0 \times$ $10^{14}$ ions per $\mathrm{cm}^{2}, 1.0 \times 10^{15}$ ions per $\mathrm{cm}^{2}$ and $5.0 \times 10^{15}$ ions per $\mathrm{cm}^{2}$ ). The mean values of erbium concentration depth distributions in the as-implanted samples were detected at a depth of 41-44 $\mathrm{nm}$ below the surface and were in a good agreement with the SRIM simulation.

Fig. 5b compares the Raman spectra of the prepared samples measured in the range of $500-1800 \mathrm{~cm}^{-1}$. Depth analysis by means of Raman spectroscopy is limited by the penetration depth of the laser light in the solid and thus depends on both its wavelength and on the extinction coefficient of the material under investigation. It means that Raman spectroscopy should be both the surface probing technique for analysing graphite and the bulk probing technique for analysing diamond. ${ }^{52}$ For example, resolving a thin glassy carbon layer on top of the perfect crystalline diamond film is limited, and similarly a low amount of diamond crystals embedded in a non-diamond carbon matrix is hardly resolvable. ${ }^{53}$ On the other hand, small amounts of graphitic material are easily detected in a diamond matrix due to the higher Raman scattering cross-section of graphite. ${ }^{52}$ The single-crystal diamond structure has a single peak at $1332 \mathrm{~cm}^{-1}$. The observed broad band at approx. $1400-1450 \mathrm{~cm}^{-1}$ (see the inset in Fig. 5b) can be attributed to the damaged surface layer of implanted diamond samples, i.e. represents either transpolyacetylene chains at diamond grain boundaries or disordered $\mathrm{sp}^{2}$ bonded carbon within the damaged diamond sub-surface. ${ }^{54}$ The higher ion implantation fluence resulted in the higher intensity of this band at around $1425 \mathrm{~cm}^{-1}$.

The RBS/channeling measurements showed that the relative numbers of disordered atoms $N_{\mathrm{D}} / N$ were about $46 \%, 49 \%$ and $69 \%$ for ion implantation fluences of $1.0 \times 10^{14}$ ions per $\mathrm{cm}^{2}$, 
(a)

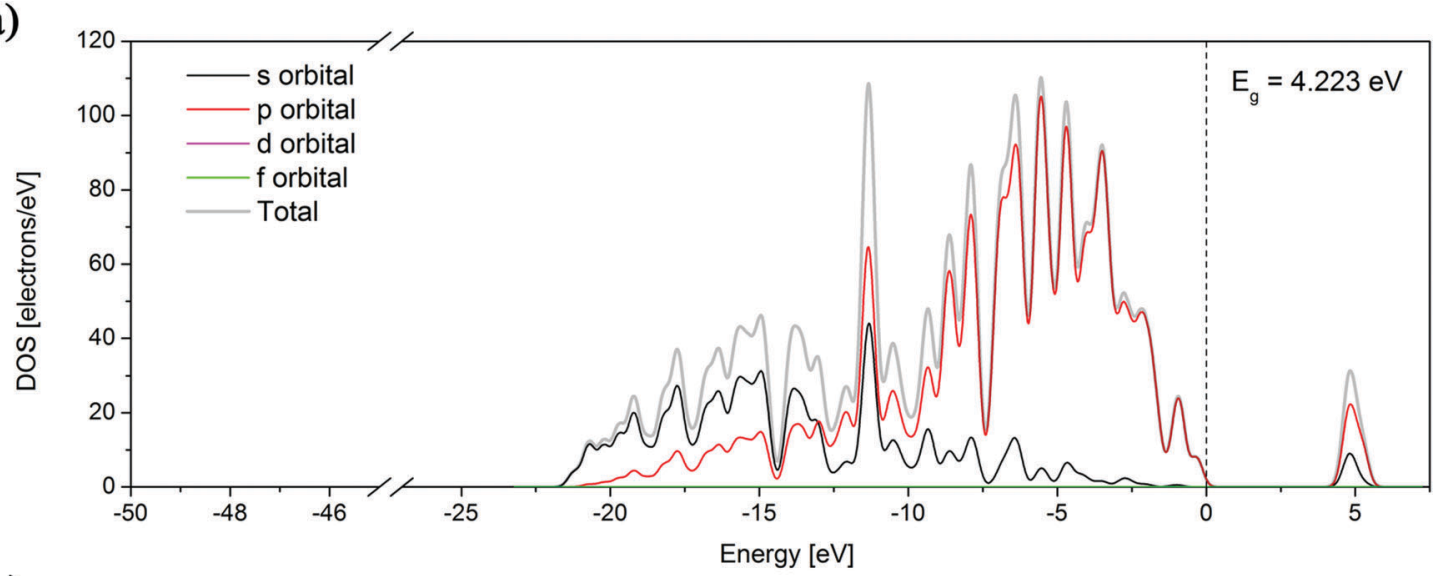

(b)

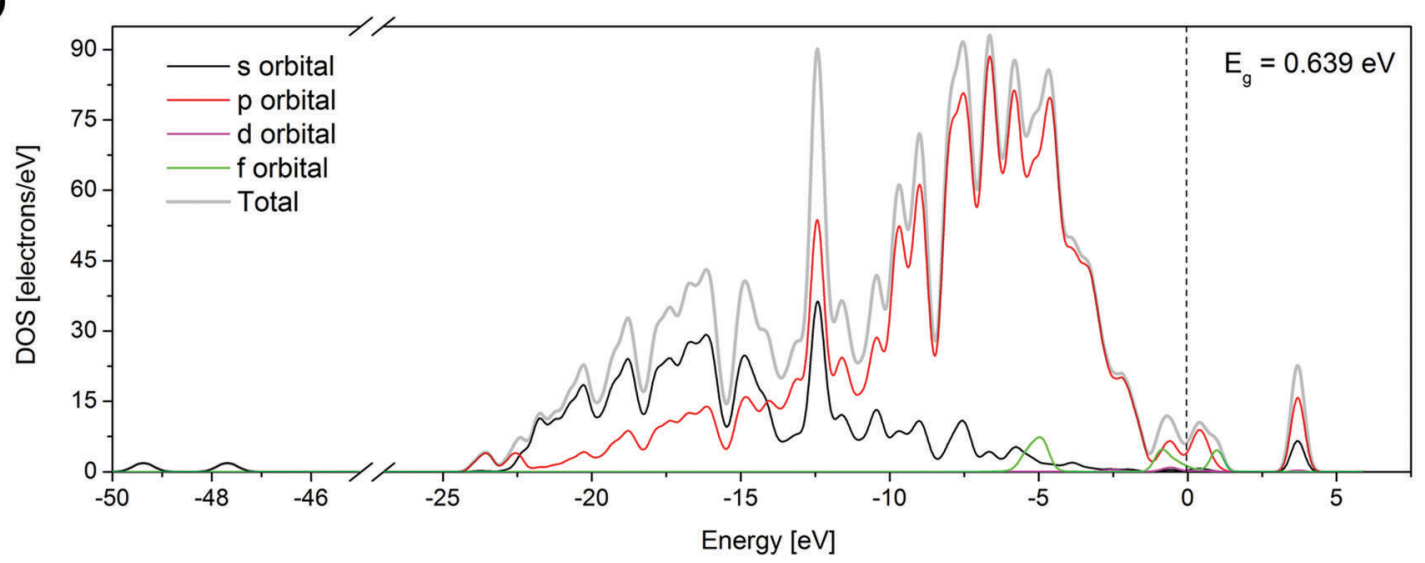

Fig. 4 Total and partial density of states for (a) parent diamond structure and (b) the most stable Er:diamond structure with Er in the substitutional position with 3 vacancies in the vicinity. The calculated band gap $\left(E_{\mathrm{g}}\right)$ values for the given structure are shown in the right-top corner of the graphs. The dotted vertical line corresponds to the Fermi level (placed at the zero level).

Table 6 The charge analysis of Er-defects in substitutional positions in the diamond structure using different charge analysis methods

\begin{tabular}{llll}
\hline Structure & Mulliken $[e]$ & Hirshfeld $[e]$ & QEq $[e]$ \\
\hline $\mathrm{Er}_{\text {sub }}:$ diamond (0 vacancies) & 3.72 & 0.32 & 0.884026 \\
$\mathrm{Er}_{\text {sub }}$ diamond (1 vacancy) & 3.38 & 0.32 & 0.884140 \\
$\mathrm{Er}_{\text {sub }}$ :diamond (2 vacancies) & 3.04 & 0.29 & 0.884228 \\
$\mathrm{Er}_{\text {sub }}$ :diamond (3 vacancies) & 2.86 & 0.39 & 0.884224 \\
$\mathrm{Er}_{\text {sub }}$ :diamond (4 vacancies) & 2.58 & 0.32 & 0.884217
\end{tabular}

Table 7 An overview of the details about prepared Er-doped diamond samples specifying the conditions of the preparation and the results of the structural analysis

\begin{tabular}{llllll}
\hline $\begin{array}{l}\text { Cut } \\
\text { (sample no.) }\end{array}$ & $\begin{array}{l}\text { Ion implantation } \\
\text { conditions }\end{array}$ & $\begin{array}{l}R_{\mathrm{p}} \\
{[\mathrm{nm}]}\end{array}$ & $\begin{array}{l}\Delta R_{\mathrm{p}} \\
{[\mathrm{nm}]}\end{array}$ & $\begin{array}{l}N_{\mathrm{D}} / N \\
{[\%]}\end{array}$ & $\begin{array}{l}\chi_{\mathrm{D}} \\
{[\%]}\end{array}$ \\
\hline $\begin{array}{l}\text { SRIM } \\
\text { Parent diamond }\end{array}$ & $\mathrm{Er}^{+}, 190 \mathrm{keV}$ & 45 & 15 & - & - \\
$Z(\mathrm{I}-277)$ & - & - & - & - & 13 \\
& $\begin{array}{l}\mathrm{Er}^{+}, 190 \mathrm{keV}, \\
Z(\mathrm{I}-278)\end{array}$ & 44 & 9 & 46 & 53 \\
& $\begin{array}{l}1.0 \times 10^{14} \text { ions per cm } \\
\mathrm{Er}^{+}, 190 \mathrm{keV},\end{array}$ & 41 & 12 & 49 & 56 \\
$Z(\mathrm{I}-279)$ & $\begin{array}{l}1.0 \times 10^{15} \text { ions per } \mathrm{cm}^{2} \\
\mathrm{Er}^{+}, 190 \mathrm{keV},\end{array}$ & 44 & 12 & 69 & 73 \\
& $5.0 \times 10^{15}$ ions per $\mathrm{cm}^{2}$ & & & & \\
& & & & &
\end{tabular}

$1.0 \times 10^{15}$ ions per $\mathrm{cm}^{2}$ and $5.0 \times 10^{15}$ ions per $\mathrm{cm}^{2}$, respectively (see Table 7). Because of the high yields of backscattered ions in the aligned spectra along the chosen crystallographic axes, the RBS/channeling method is very sensitive to the number of structural disordered atoms; therefore, the structural changes in the implanted samples were examined using this method. The measured random and aligned spectra of all three samples are shown in Fig. 6. It follows from these measurements that the density of the disordered atoms - i.e. $N_{\mathrm{D}} / N$ - is approximately $50-70 \%$ for the samples doped with increasing ion implantation fluence. The fractions of the implanted erbium ions entering substitutional positions after the ion implantation were determined from the change in the area of the erbium depth profiles. At the ion implantation fluences used, no significant preferential substitutional position was observed after ion implantation. It means that a negligible amount of about $5-10 \%$ of erbium ions was found in substitutional positions while other erbium atoms were in interstitial positions. Fine scans were carried out, but due to the high degree of structural damage it was impossible to determine the erbium positions in the structure. This effect was expected, as the preferential positioning of the dopant in the highly disordered structure is in fact impossible.

The measured luminescence spectra of $\mathrm{X}$ and $\mathrm{Z}$ cut Er:diamond samples non-annealed and annealed at the temperatures of $600{ }^{\circ} \mathrm{C}$ and $800{ }^{\circ} \mathrm{C}$ in a vacuum are shown in Fig. 7. The $\mathrm{X}$ cut was doped with the fluence of $1.0 \times 10^{15}$ ions per $\mathrm{cm}^{2}$ and the $\mathrm{Z}$ cut was 
(a)

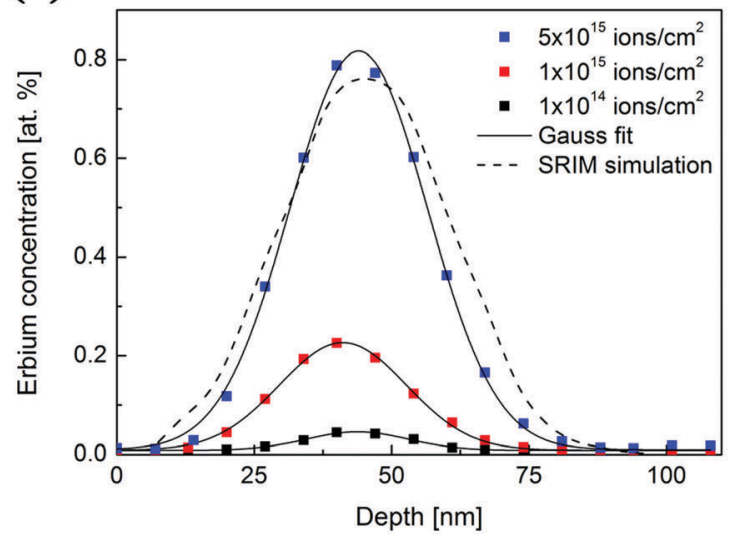

(b)

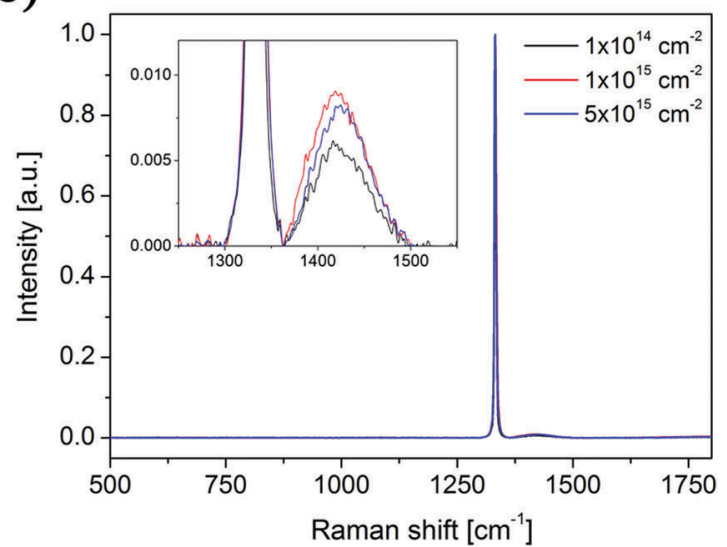

Fig. 5 (a) The erbium concentration depth profiles of the prepared Z-cut samples of Er:diamond using different ion implantation fluences. (b) Normalized Raman spectra of the implanted diamond samples. The zoomed spectral region for the non-diamond-based band is shown in the inset.

(a)

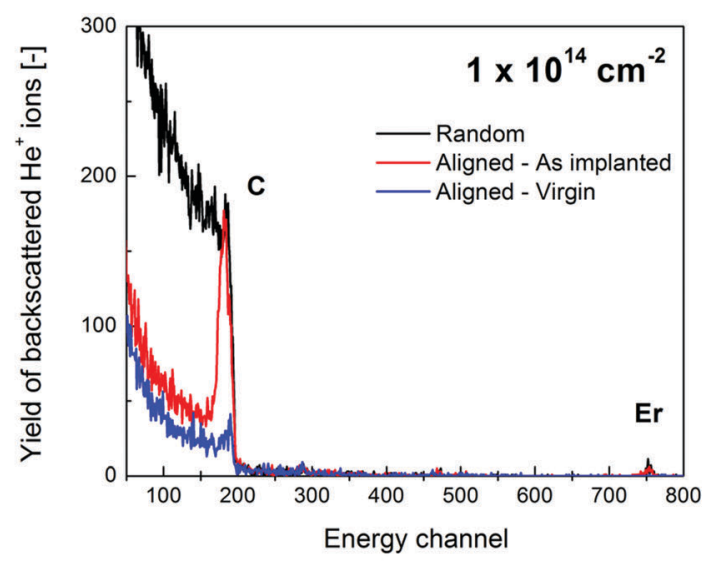

(b)

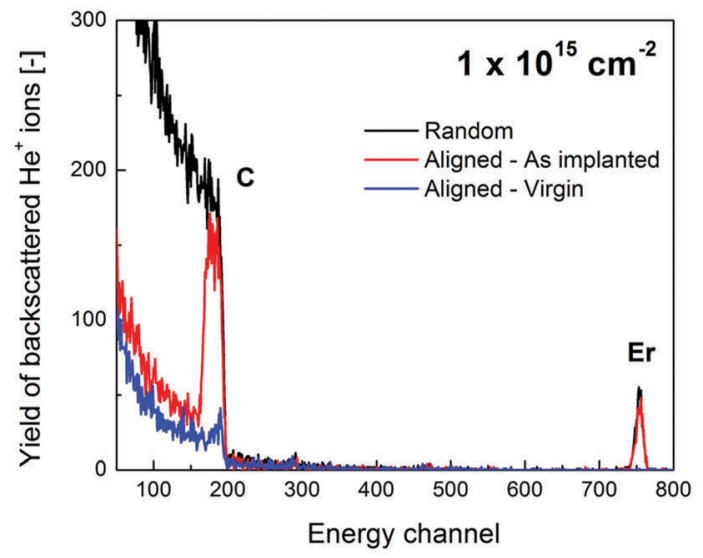

(c)

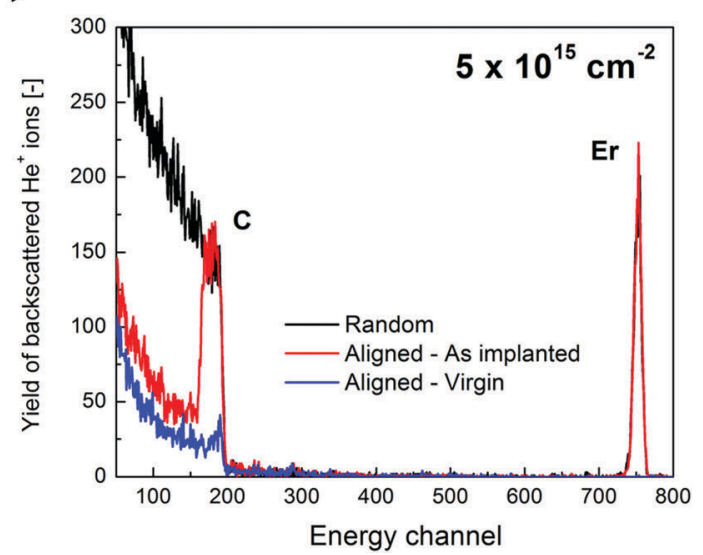

Fig. 6 The measured RBS/channelling spectra of Er:diamond samples doped with the ion implantation fluences of (a) $1.0 \times 10^{14}$ ions per $\mathrm{cm}^{2}$, (b) $1.0 \times 10^{15}$ ions per $\mathrm{cm}^{2}$ and (c) $5.0 \times 10^{15}$ ions per $\mathrm{cm}^{2}$.

doped with the fluences of $1.0 \times 10^{14}$ ions per $\mathrm{cm}^{2}, 1.0 \times 10^{15}$ ions per $\mathrm{cm}^{2}$ and $5.0 \times 10^{15}$ ions per $\mathrm{cm}^{2}$. All the shown spectra were baseline-corrected to the standard sample and it is therefore able to compare the luminescence intensities of different samples in the spectra. From the luminescence spectra it can be seen that all the samples revealed two main luminescence bands at around 1503 and $1535 \mathrm{~nm}$, whereas the $\mathrm{X}$ cut also had two additional luminescence bands at around 1561 and $1585 \mathrm{~nm}$. All the observed 
(a)

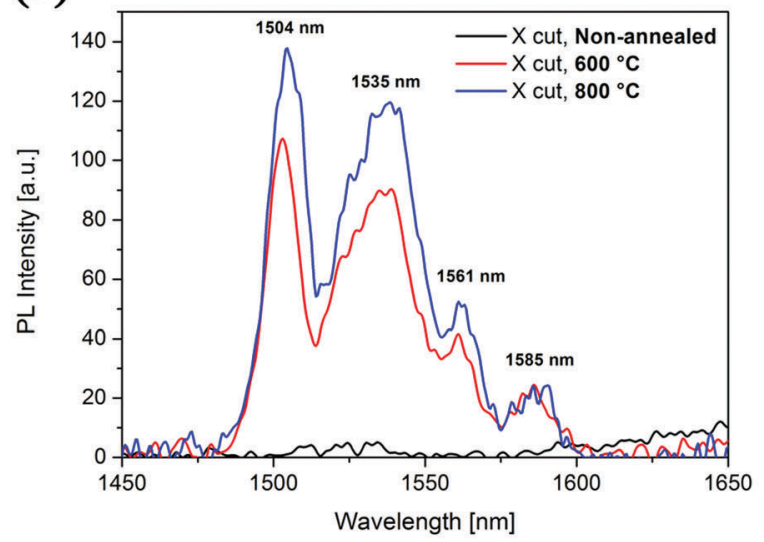

(b)

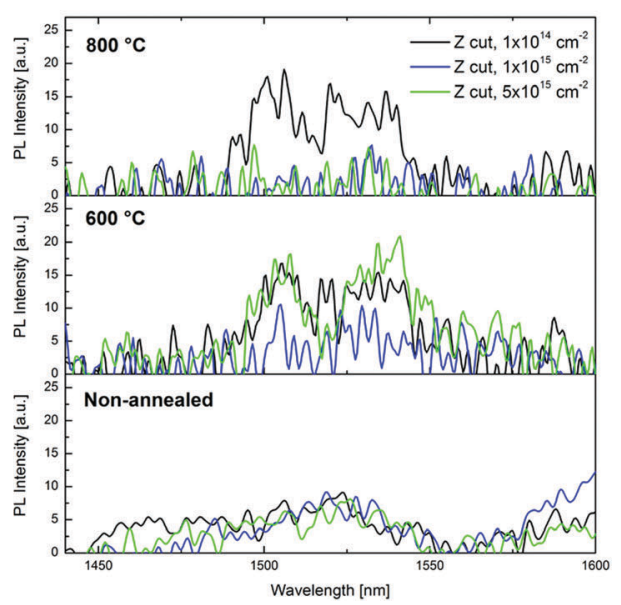

Fig. 7 The measured luminescence spectra of Er-doped diamond samples non-annealed and annealed at $600{ }^{\circ} \mathrm{C}$ and $800{ }^{\circ} \mathrm{C}$ for (a) $\mathrm{X}$ cut doped with the fluence of $1.0 \times 10^{15}$ ions per $\mathrm{cm}^{2}$ and (b) Z cut doped with the fluences of $1.0 \times 10^{14}$ ions per $\mathrm{cm}^{2}, 1.0 \times 10^{15}$ ions per $\mathrm{cm}^{2}$ and $5.0 \times 10^{15}$ ions per $\mathrm{cm}^{2}$.

luminescence bands correspond to the ${ }^{4} \mathrm{I}_{11 / 2} \rightarrow{ }^{4} \mathrm{I}_{15 / 2}$ electronic transitions of Er ions. The luminescence intensities of the $\mathrm{Z}$ cut samples were overall very low as compared to the $\mathrm{X}$ cut, i.e. the $\mathrm{X}$ cut had approximately 6-7 times higher luminescence intensity than $\mathrm{Z}$ cut samples. The highest-intensity luminescence in the $\mathrm{Z}$ cut was achieved for the lowest used ion implantation fluence of $1.0 \times 10^{14}$ ions per $\mathrm{cm}^{2}$ annealed at $800{ }^{\circ} \mathrm{C}$. Measurements of the luminescence spectra of the non-annealed and $400{ }^{\circ} \mathrm{C}$ annealed samples showed practically no measurable luminescence for both $\mathrm{X}$ and $\mathrm{Z}$ cuts. Annealing of the $\mathrm{X}$ cut in a vacuum caused a substantial increase of the luminescence intensity - after the annealing at $400{ }^{\circ} \mathrm{C}$ no measurable luminescence was achieved, however after the annealing at $600{ }^{\circ} \mathrm{C}$ a substantial increase in luminescence was achieved with 4 pronounced luminescence peaks and the annealing at $800{ }^{\circ} \mathrm{C}$ only slightly increased $(\mathrm{ca}$. $20-30 \%$ ) the luminescence intensity while causing no further change to the spectra.

The theoretical and experimental results can be summarized as follows:

(1) According to the theoretical model erbium in the diamond structure occupies substitutional positions rather than interstitial ones. The incorporation of erbium into the diamond structure does not invoke any serious structural changes or broken bonds according to the calculations. The main reason why the substitutional carbon position is more preferred than the interstitial position can be seen when looking on the geometry optimization steps for both structural models where the ability to the relaxation of the structure is a key issue. The interstitial tetrahedral vacancy position has almost no degree of freedom to relax - all the carbon atoms around the vacancy are covalently bonded. On the other hand, when the carbon vacancy is formed, there are several carbon atoms in its vicinity that are just partly bonded (to 3 other carbon atoms) in the diamond structure and therefore are able to move with certain degrees of freedom making more space for the Er atom when incorporated. Therefore, as a result of the model, the structure is more stable if one to three vacant positions are formed in the surroundings of erbium and erbium is displaced in the octahedral vacancy.
(2) Experimental results showed that using the ion implantation doping method it is possible to enrich diamond with erbium with the concentrations from 800 to $8000 \mathrm{ppm} .800 \mathrm{ppm}$ is a value that is mostly found in the materials currently used in photonic amplifying devices (e.g. in the glass fibres there is usually about $500 \mathrm{ppm}$ of erbium, i.e. 0.05 at\%). This amount, which does not dramatically increase the optical losses, can be achieved using the ion implantation fluence of $1 \times 10^{14}$ ions per $\mathrm{cm}^{2}$. However, the diamond structure is strongly damaged in the ion implantation spot of incidence, it means that 46 to 60 percent of carbon atoms are shifted from their original positions. From this amount only 5 to 10 percent of erbium atoms are situated in the substitutional positions. We found a significantly higher structural damage in the samples implanted by $1 \times 10^{15}$ ions per $\mathrm{cm}^{2}$ as compared with the fluence of $1 \times 10^{14}$ ions per $\mathrm{cm}^{2}$. This fact was also confirmed by Raman spectroscopy measurements. According to the luminescence measurement it turned out that the postimplantation annealing has a beneficial effect on the luminescence, where after the annealing at the temperature of $800{ }^{\circ} \mathrm{C}$ a substantial increase of the luminescence intensity was achieved for the $\mathrm{X}$ cut of Er doped diamond samples.

\section{Discussion}

In the theoretical model, the calculated values of cohesive energies were compared with the experimental ones for pure diamond yielding a very good agreement. The calculation of the band gap of pure diamond is in a good agreement with the previous study of doping of oxygen in diamond ${ }^{25}$ indicating the good choice of the used DFT functional. Concerning the diamond doping, all the published results obtained by the DFT models dealing with positions of various elements in the diamond structure implicate that the vast majority of used dopants are located in the substitutional carbon sites. ${ }^{23-27}$ From this point of view it is not surprising that our model involving a large set of atoms predicts the preferential substitutional carbon position for erbium atoms. The only similar study of the doping of lanthanide 
atoms in the diamond structure was done by the group of $\mathrm{A}$. Magyar et $a l^{23}$ simulating europium atoms in diamond with a variable number of carbon vacancies and finding the most stable configuration which turned out to be $\mathrm{Eu}$ in the substitutional position with one carbon vacancy in the Eu vicinity. This is in a good agreement with our results. The values of defect formation energies for $\mathrm{Er}$ in the substitutional position with 1-3 vacancies are comparable with the values calculated for europium. The lowest defect formation energies are around $11 \mathrm{eV}$ for both lanthanides ( $\mathrm{Eu}$ and $\mathrm{Er}$ ). Moreover, from our theoretical results for the Er in the substitutional position the three vacancies are even more preferred than one vacancy. We also found that the interstitial position is less favourable than the substitutional carbon position, irrespective of the number of surrounding C-vacancies involved. Obviously the charge balance in the DFT model is rather uncertain. For erbium in the diamond structure with one or three vacancies in its neighbourhood the most probable charge of erbium was calculated to be +0.32 and +0.39 , respectively, from the Hirshfeld analysis. This result also corresponds to the value reported in ref. 22 , where the europium charge lies between 0 and +1 . The details of the band structure around the Fermi level formed by Er-4f states were also analysed in this study. The width of the band gap of Er-doped diamond was lowered to $0.639 \mathrm{eV}$. A similar lowering was also observed for the $\mathrm{Eu}$ and $\mathrm{O}$ atoms in the diamond structure. ${ }^{23,25}$

Ion implantation of various elements into the diamond structure has already been a subject of many studies, e.g. the incorporation of N, Si, Ni, Cr, C, Ce, Gd, He and others. ${ }^{2,22,55-57}$ In these studies various implantation fluences $\left(1 \times 10^{10}-1 \times\right.$ $10^{17}$ ions per $\mathrm{cm}^{2}$ ) as well as a large range of ion implantation energies (30 keV-2.5 MeV) have been used. The main intention was to form less-defective structures containing a lower concentration of dopants. In our experiments we have intentionally chosen higher fluences, motivated by our previous experience with luminescence measurements of erbium implanted materials using different implantation fluences. ${ }^{58}$ It is known that in contrast to oxide materials the recovery of the diamond structure is a quite difficult process since the structure tends to amorphise. According to ref. 59 the critical defect density, after which the damaged structure cannot be recovered back to the original diamond structure, is $1 \times 10^{22}$ vacancies per $\mathrm{cm}^{3}$ (using $52 \mathrm{eV}$ for the displacement energy). It can be seen from the Raman spectra of our samples (Fig. $5 \mathrm{~b}$ ) that a fluence of $1 \times 10^{14}$ ions per $\mathrm{cm}^{2}$ yielded a much lower amount of amorphous carbon (according to the broad peak at around 1400-1450 $\mathrm{cm}^{-1}$ ) in comparison with the other two higher fluences. We therefore suppose that partial healing of this damaged structure can be achieved. Higher fluences however would mean much more serious damage to the structure. Regarding the fact that erbium, being an f-element, is little influenced by the surrounding crystal field in the matrix material due to the shielding of f-orbitals by outer $5 \mathrm{~s}$ and $5 \mathrm{p}$ orbitals, it is by far more important to avoid any non-radiative transitions (from e.g. clustering of atoms) and to have suitable oxidation states of the atoms in the erbium vicinity. This fact brings us to a conclusion that we can expect a reasonably high luminescence even though a part of the diamond sample might be amorphised. In contrast, the defects in the amorphised structure (such as grain boundaries in the NCD diamond) may be even beneficial for the separation of the erbium ions and consequently the higher measurable luminescence.

\section{Conclusions}

In the present study a theoretical modelling approach was applied to assess the point defect energies in the erbium doped single-crystal diamond structure and compared with the experimental results from the erbium ion implantation into the $\mathrm{Z}$ cut $\langle 001\rangle$ of the single-crystal diamond. The calculated cohesive energy of the parent diamond structure was in a very good agreement with the experimental value, having a difference of approximately $1.0 \%$. According to the theoretical modelling results it turned out that erbium is able to exist in both substitutional and interstitial diamond positions without serious parent-diamond structural changes or bond breakage. Comparing the cohesive and the defect formation energies of the respective structural models, it is found that a more probable erbium location in diamond is the substitutional site of carbon with one or three vacancies in the vicinity of erbium.

The ion implantation process caused a substantial damage to the structure of the implanted layers involving up to $69 \%$ of atoms being disordered, which prevented the determination of the absolute erbium positions in the crystallographic structure. The prepared Er-doped diamond samples annealed at the temperatures of 400,600 and $800{ }^{\circ} \mathrm{C}$ in a vacuum revealed clear luminescence, whereas the $\mathrm{X}$ cut $\langle 110\rangle$ sample has approximately 6-7 times higher luminescence intensity than the $\mathrm{Z}$ cut $\langle 001\rangle$ sample with the same ion implantation fluence. The reported results are to our knowledge the first demonstration of the Er luminescence in the single crystal diamond structure for the near-infrared spectral region.

\section{Acknowledgements}

We acknowledge the Czech Science Foundation project GA 1405053S and financial support from specific university research (MSMT No. 20/2015). Some parts of the research were realised at the CANAM (Center of Accelerators and Nuclear Analytical Methods LM 2015056) infrastructure. This work was also supported by the MSMT project No. LD15003 (MV, AK) within the frame of the COST Action MP1403 "Nanoscale Quantum Optics".

\section{References}

1 A. G. A. Grill, Diamond-like carbon coatings as biocompatible materials - an overview, Diamond Relat. Mater., 2003, 12, 166.

2 J. F. Prins, Implantation-doping of diamond with $\mathrm{B}+, \mathrm{C}+, \mathrm{N}+$ and $\mathrm{O}+$ ions using low temperature annealing, Diamond Relat. Mater., 2002, 11, 612.

3 N. Tsubouchi, M. Ogura and H. Kato, et al., p-type doping by $\mathrm{B}$ ion implantation into diamond at elevated temperatures, Diamond Relat. Mater., 2006, 15, 157. 
4 R. Kalish, The search for donors in diamond, Diamond Relat. Mater., 2001, 10, 1749.

5 T. Sharda, T. Soga and T. Jimbo, Optical properties of nanocrystalline diamond films by prism coupling technique, J. Appl. Phys., 2003, 93, 101.

6 C. Manolatou, S. G. Johnson, S. H. Fan, P. R. Villeneuve, H. A. Haus and J. D. Joannopoulos, High-density integrated optics, J. Lightwave Technol., 1999, 17, 1682-1692.

7 B. E. Little, J. S. Foresi, G. Steinmeyer, E. R. Thoen, S. T. Chu, H. A. Haus, E. P. Ippen, L. C. Kimerling and W. Greene, Ultracompact $\mathrm{Si}_{-} \mathrm{SiO}_{2}$ microring resonator optical channel dropping filters, IEEE Photonics Technol. Lett., 1998, 10, 549-551.

8 V. Prajzler, P. Nekvindova, M. Varga, J. Bruncko, Z. Remes and A. Kromka, Prism coupling technique for characterization of the high refractive index planar waveguides, J. Optoelectron. Adv. Mater., 2016, 18, 915-921.

9 I. Aharonovich and E. Neu, Diamond nanophotonics, Adv. Opt. Mater., 2014, 2(10), 911-928.

10 J. O. Orwa, A. D. Greentree and I. Aharonovich, et al., Fabrication of single optical centres in diamond-a review, J. Lumin., 2010, 130, 1646.

11 A. Gruber, A. Dräbenstedt, C. Tietz, L. Fleury, J. Wrachtrup and C. Von Borczyskowski, Scanning confocal optical microscopy and magnetic resonance on single defect centers, Science, 1997, 276(5321), 2012-2014.

12 A. Beveratos, S. Kühn, R. Brouri, T. Gacoin, J. P. Poizat and P. Grangier, Room temperature stable single-photon source, Eur. Phys. J. D, 2002, 18(2), 191-196.

13 C. D. Clark, H. Kanda, I. Kiflawi and G. Sittas, Silicon defects in diamond, Phys. Rev. B: Condens. Matter Mater. Phys., 1995, 51(23), 16681.

14 T. Gaebel, I. Popa, A. Gruber, M. Domhan, F. Jelezko and J. Wrachtrup, New J. Phys., 2004, 6, 98-104.

15 I. Aharonovich, S. Castelletto, D. A. Simpson, A. Stacey, J. McCallum, A. D. Greentree and S. Prawer, Two-level ultrabright single photon emission from diamond nanocrystals, Nano Lett., 2009, 9(9), 3191-3195.

16 C. P. Foy, M. C. do Carmo, G. Davies and E. C. Lightowlers, Uniaxial stress measurements on the $0.97 \mathrm{eV}$ line in irradiated silicon, J. Phys. C: Solid State Phys., 1981, 14(1), L7.

17 I. Aharonovich, S. Castelletto, D. A Simpson, C. H. Su, A. D. Greentree and S. Prawer, Diamond-based single-photon emitters, Rep. Prog. Phys., 2011, 74(7), 076501.

18 D. W. M. Lau, T. J. Karle, B. C. Johnson, B. C. Gibson, S. Tomljenovic-Hanic, A. D. Greentree and S. Prawer, Very bright, near-infrared single photon emitters in diamond, APL Mater., 2013, 1(3), 032120.

19 S. Castelletto, A. Edmonds, T. Gaebel and J. Rabeau, Production of multiple diamond-based single-photon sources, IEEE J. Sel. Top. Quantum Electron., 2012, 18(6), 1792-1798.

20 I. I. Vlasov, O. Shenderova, S. Turner, O. I. Lebedev, A. A. Basov, I. Sildos and G. Van Tendeloo, Nitrogen and Luminescent Nitrogen-Vacancy Defects in Detonation Nanodiamond, Small, 2010, 6(5), 687-694.

21 E. Ōsawa, Recent progress and perspectives in single-digit nanodiamond, Diamond Relat. Mater., 2007, 16(12), 2018-2022.
22 K. Bharuth-Ram, U. Vetter, H. Hofsäss, C. Ronning, M. Dietrich and Isolde Collaboration, Implantation sites of Ce and Gd in diamond, Nucl. Instrum. Methods Phys. Res., Sect. B, 2002, 190(1), 835-839.

23 A. Magyar, W. Hu and T. Shanley, et al., Synthesis of luminescent europium defects in diamond, Nat. Commun., 2014, 5, 3523.

24 C. X. Yan, Y. Dai and B. B. Huang, DFT study of halogen impurity in diamond, J. Phys. D: Appl. Phys., 2009, 42(14), 145407.

$25 \mathrm{M}$. Ullah, An investigation into the properties of nano crystalline diamond thin films grown by HFCVD system, PhD thesis, University Multan, Pakistan, 2013.

26 M. Ullah, E. Ahmed, F. Hussain, A. M. Rana and R. Raza, Electrical conductivity enhancement by boron-doping in diamond using first principle calculations, Appl. Surf. Sci., 2015, 334, 40-44.

27 M. Ullah, E. Ahmed, F. Hussain, A. M. Rana, R. Raza and H. Ullah, Electronic structure calculations of oxygen-doped diamond using DFT technique, Microelectron. Eng., 2015, 146, 26-31.

28 R. Kalish, A. Reznik, S. Prawer, D. Saada and J. Adler, IonImplantation-Induced Defects in Diamond and Their Annealing: Experiment and Simulation, Phys. Status Solidi A, 1999, 174(1), 83-99.

29 S. J. Clark, M. D. Segall, C. J. Pickard, P. J. Hasnip, M. J. Probert, K. Refson and M. C. Payne, First principles methods using CASTEP, Z. Kristallogr., 2005, 220(5-6), 567-570.

30 A. K. Rappe and W. A. Goddard, Charge equilibration for molecular dynamics simulations, J. Phys. Chem., 1991, 95(8), 3358-3363.

31 B. Winkler, C. J. Pickard, M. D. Segall and V. Milman, Density functional study of charge ordering in $\mathrm{Cs}_{2} \mathrm{Au}(\mathrm{I}) \mathrm{Au}(\mathrm{III}) \mathrm{Cl}_{6}$ under pressure, Phys. Rev. B: Condens. Matter Mater. Phys., 2001, 63, 14103.

32 M. D. Segall, R. Shah, C. J. Pickard and M. C. Payne, Population analysis of plane-wave electronic structure calculations of bulk materials, Phys. Rev. B: Condens. Matter Mater. Phys., 1996, 54, 16317-16320.

33 R. S. Mulliken, J. Chem. Phys., 1955, 23, 1833-1846.

34 F. L. Hirshfeld, Bonded-Atom Fragments for Describing Molecular Charge Densities, Theor. Chim. Acta, 1977, 44, 129-138.

35 J. Saarilahti and E. Rauhala, Interactive personal-computer data analysis of ion backscattering spectra, Nucl. Instrum. Methods Phys. Res., Sect. B, 1992, 64, 734.

36 M. Mayer, SIMNRA version 6.06, Max-Planck-Institut fur Plasmaphysik, Garching, Germany, 2011, http://home.rzg. mpg.de/ mam/.

37 IBANDL, http://www-nds.iaea.org/ibandl/GISA.

38 H. Shin, S. Kang and J. Koo, et al., Cohesion Energetics of Carbon Allotropes: Quantum Monte Carlo Study, J. Chem. Phys., 2014, 114702.

39 C. Kittel, Introduction to Solid State Physics, Wiley, 1996.

40 G. M. D. Nguimdoa and D. P. Joubert, A density functional (PBE, PBEsol, HSE06) study of the structural, electronic and optical properties of the ternary compounds $\operatorname{AgAlX}_{2}(X=S$, Se, Te), Eur. Phys. J., 2015, 88, 113. 
41 H.-P. Komsa, T. Rantala and A. Pasquarello, Comparison between various finite-size supercell correction schemes for charged defect calculations, Physica B, 2012, 407, 3063-3067.

42 Y. Li, W. G. Schmidt and S. Sanna, Defect complexes in congruent $\mathrm{LiNbO}_{3}$ and their optical signatures, Phys. Rev. B: Condens. Matter Mater. Phys., 2015, 91, 174106.

43 Y. Li, W. G. Schmidt and S. Sanna, Intrinsic $\mathrm{LiNbO}_{3}$ point defects from hybrid density functional calculations, Phys. Rev. B: Condens. Matter Mater. Phys., 2014, 89, 094111.

44 R. D. Shannon, Revised Effective Ionic Radii and Systematic Studies of Interatomic Distances in Halides and Chalcogenides, Acta Crystallogr., Sect. A: Cryst. Phys., Diffr., Theor. Gen. Crystallogr., 1976, 32, 751-767.

45 From tabulations and averages of $\mathrm{C}\left(\mathrm{sp}^{3}\right)-\mathrm{X}$ distances in: F. H. Allen, O. Kennard, D. G. Watson, L. Brammer, A. G. Orpen and R. Taylor, J. Chem. Soc., Perkin Trans. 2, 1987, S1-S19, DOI: 10.1039/P298700000S1.

46 D. N. Singh, Basic Concepts Of Inorganic Chemistry, Pearson, India, 2010, ISBN: 978-8131728109.

47 X.-H. Hua, J.-H. Xue, L.-M. Yang, Y.-Z. Xu and J.-G. Wua, Butane-1,2,3,4-tetraol- $\left(\kappa^{3} O^{1} O^{2} O^{3}\right)($ ethanol- $\kappa O)$ tris(nitrato- $\left.\kappa^{2} O O^{\prime}\right)$ erbium(III), Acta Crystallogr., Sect. E: Struct. Rep. Online, 2013, 69(5), 257-258.

48 B. Gyurcsik and L. Nagy, Carbohydrates as ligands: coordination equilibria and structure of the metal complexes, Coord. Chem. Rev., 2000, 203, 81-149.

49 L.-M. Yang, X.-H. Hua, J.-H. Xue, Q.-H. Pan, L. Yu, W.-H. Li, Y.-Z. Xu, G.-Z. Zhao, L.-M. Liu, K.-X. Liu, J.-E. Chen and J.-G. Wu, Interactions between Metal Ions and Carbohydrates. Spectroscopic Characterization and the Topology Coordination Behaviour of Erythritol with Trivalent Lanthanide Ions, Inorg. Chem., 2012, 51, 499-510.
50 "CODATA Value: elementary charge", The NIST Reference on Constants, Units, and Uncertainty. US National Institute of Standards and Technology, June 2015, Retrieved 2016-12-10.

51 J. R. Tesmer and M. A. Nastasi, Handbook of Modern Ion Beam Analysis, Materials Research Society, Michigan, 1995.

52 R. E. Clausing, L. L. Horton, J. C. Angus and P. Koidl, Diamond and Diamond-like Films and Coatings, Springer, USA, Boston, 1991.

53 M. Varga, T. Izak, V. Vretenar, H. Kozak, J. Holovsky, A. Artemenko, M. Hulman, V. Skakalova, D. S. Lee and A. Kromka, Diamond/carbon nanotube composites: Raman, FTIR and XPS spectroscopic studies, Carbon, 2017, 111, 54-61.

54 F. Klauser, D. Steinmüller-Nethl, R. Kaindl, E. Bertel and N. Memmel, Raman Studies of Nano- and Ultra-nanocrystalline Diamond Films Grown by Hot-Filament CVD, Chem. Vap. Deposition, 2010, 16, 127-135.

55 S. Castelletto, I. Aharonovich, B. C. Gibson, B. C. Johnson and S. Prawer, Imaging and quantum-efficiency measurement of chromium emitters in diamond, Phys. Rev. Lett., 2010, 105(21), 217403.

56 L. Himics, S. Toth, M. Veres, A. Toth and M. Koos, Effective implantation of light emitting centers by plasma immersion ion implantation and focused ion beam methods into nanosized diamond, Appl. Surf. Sci., 2015, 328, 577-582.

57 P. F. Lai, S. Prawer and L. A. Bursill, Recovery of diamond after irradiation at high energy and annealing, Diamond Relat. Mater., 2001, 10(1), 82-86.

58 P. Nekvindova, B. Svecova and J. Cajzl, et al., Erbium ion implantation into different crystallographic cuts of lithium niobate, Opt. Mater., 2011, 34, 652-659.

59 C. Uzan-Saguy, C. Cytermann, R. Brener, V. Richter, M. Shaanan and R. Kalish, Damage threshold for ion-beam induced graphitization of diamond, Appl. Phys. Lett., 1995, 67(9), 1194-1196. 